\title{
Existence of second order smooth solutions for 2D Euler equations with symmetry outside a core region
}

\author{
Yanyun Wu' ${ }^{1}$ Liquan $\mathrm{Mei}^{1^{*}}$ and Ganshan Yang ${ }^{2}$
}

\section{${ }^{\text {"Correspondence: }}$}

lqmei@mail.xjtu.edu.cn

'School of Mathematics and

Statistics, Xi'an Jiaotong University, Xi'an, 710049, China

Full list of author information is

available at the end of the article

\section{照 Springer}

\begin{abstract}
In this paper, we study the existence of second order smooth solutions for the initial boundary value problem of Euler equation satisfying the $\gamma$-law with damping and axial symmetry. After a series of transformations, we can choose the $\gamma$ in an appropriate scope to make sure the estimates of the $C^{2}$ norm of the solutions are bounded when the damping is strong enough.
\end{abstract}

MSC: $35 \mathrm{~A} 01 ; 76 \mathrm{~N} 10$

Keywords: characteristic method; Young inequality; Gronwall inequality; $C^{2}$ norm; second order smooth solution

\section{Introduction}

The Euler equation is one of the most important fundamental equations in inviscid fluid dynamics, which describes the motion of a fluid in $\mathbb{R}^{d}$. The existence, uniqueness, and the regularity of solutions of Euler equations are three popular research topics. In the case of periodic boundary conditions and for arbitrary dimension $d \geq 2$, there exist infinitely many global weak solutions for the incompressible Euler equation with initial data [1]. Zhang and Zheng [2] constructed a two parameter family of self-similar solutions to both the compressible and the incompressible 2D Euler equations with axisymmetry. They also provided the vortical and self-similar solutions for 2D compressible Euler equations using a separation method [3]. The stationary classical solutions of the incompressible Euler equation approximating singular stationary solutions of this equation have been constructed in [4]. The global existence and uniqueness of the classical solutions to the initial boundary value problem for the 3D damped compressible Euler equation have been proved on a bounded domain with slip boundary condition when the initial data is near its equilibrium [5]. A smooth solution of the 2D Euler equation on a bounded domain exists and is unique in a natural class locally in time, but it blows up in finite time in the sense of its vorticity losing continuity [6]. There are also some exact blowup solutions to the pressureless Euler equations [7]. If the domain and the initial data are smooth, results on the global smooth solutions of the 2D Euler equation go back to the 1930s. Marin [8] studied the temporal behavior of the solutions of some equations which are applied in our daily life. The global existence of regular solutions for Cauchy and initial boundary value

(c) 2015 Wu et al. This article is distributed under the terms of the Creative Commons Attribution 4.0 International License (http://creativecommons.org/licenses/by/4.0/), which permits unrestricted use, distribution, and reproduction in any medium, provided you give appropriate credit to the original author(s) and the source, provide a link to the Creative Commons license, and indicate if changes were made. 
problem of one dimensional quasilinear hyperbolic systems has been extensively investigated (see [2, 3, 8-18]). There exist global smooth solutions for the 2D Euler equation with symmetry [19]. The 3D inviscid model with an appropriate Neumann-Robin boundary condition was developed with a finite time singularity in an axisymmetric domain and this model has global smooth solutions for a class of a large smooth initial data with some appropriate boundary condition [20].

The contribution of our work is a study of the second order smooth solutions for the 2D compressible isentropic Euler equation with axial symmetry and damping outside a core region. We mainly use characteristic methods. Some appropriate conditions are given which guarantee the existence of second order smooth solutions. The study is based on the technical estimation of the $C^{2}$ norm of the solution.

The rest of the paper is organized as follows. Section 2 introduces the 2D Euler equation with axial symmetry and damping and gives the conclusion that there exists one order smooth solutions for Euler equation with symmetry [19]. In Section 3, the boundedness of $C^{2}$ norm of solutions is obtained under some assumptions. In Section 4 , we get the existence of second order smooth solutions for 2D Euler equation with symmetry outside a core region.

\section{2D Euler equation with axial symmetry and damping}

Consider the 2D compressible and polytropic Euler equation with damping

$$
\left\{\begin{array}{l}
\tilde{\rho}_{t}+(\tilde{\rho} u)_{x}+(\tilde{\rho} v)_{y}=0, \\
(\tilde{\rho} u)_{t}+\left(\tilde{\rho} u^{2}+P\right)_{x}+(\tilde{\rho} u v)_{y}=-2 \tilde{\rho} \alpha u \\
(\tilde{\rho} v)_{t}+(\tilde{\rho} u v)_{x}+\left(\tilde{\rho} v^{2}+P\right)_{y}=-2 \tilde{\rho} \alpha v
\end{array}\right.
$$

where $P=K^{2} \tilde{\rho}^{\gamma}$ and the friction $\alpha>0$ is a constant. Here $\rho,(u, v), P(\rho)$ are the density, velocity, and pressure, respectively. We consider (1) with axisymmetry, i.e., we look for the solutions satisfying

$$
\begin{aligned}
& \tilde{\rho}(r, \theta)=\tilde{\rho}(r, 0), \\
& \left(\begin{array}{l}
u(r, \theta) \\
v(r, \theta)
\end{array}\right)=\left(\begin{array}{cc}
\cos \theta & -\sin \theta \\
\sin \theta & \cos \theta
\end{array}\right)\left(\begin{array}{l}
u(r, 0) \\
v(r, 0)
\end{array}\right),
\end{aligned}
$$

where $(r, \theta)$ are the polar coordinates. Under (2), (1) can be written as

$$
\begin{aligned}
& \tilde{\rho}_{t}+(\tilde{\rho} u)_{r}+\frac{\tilde{\rho} u}{r}=0, \\
& u_{t}+u u_{r}+h_{r}=-2 \alpha u+\frac{v^{2}}{r}, \\
& v_{t}+u v_{r}=-2 \alpha v-\frac{u v}{r},
\end{aligned}
$$

where $h_{r}=K^{2} \gamma \tilde{\rho}^{\gamma-2} \tilde{\rho}_{r}$ is the entropy.

In this section, we are going to study system (3) outside a core region, i.e. $1 \leq r \leq \infty$, with initial and boundary data given by

$$
\tilde{\rho}(r, 0)=\tilde{\rho}_{0}(r), \quad(u, v)(r, 0)=\left(u_{0}, v_{0}\right)(r), \quad u(1, t)=0 .
$$


According to a series of transformations (refer to [19]), we obtain

$$
\begin{aligned}
& \sigma_{t}-u_{x}=0, \\
& u_{t}+\left(\frac{K^{2}}{\sigma^{\gamma}}\right)_{x} \frac{1}{r^{\gamma-1}}=-2 \alpha u+\frac{K^{2} \gamma \sigma^{1-\gamma}}{r^{\gamma}}+\frac{v^{2}}{r}, \\
& v_{t}=-2 \alpha \nu-\frac{u \nu}{r},
\end{aligned}
$$

where $r=1+\int_{0}^{x} \sigma(s, t) d s$ and $x \geq 0$. In order to symmetrize the system (5), we introduce the Riemann invariants

$$
\begin{aligned}
& w=u+\frac{2 k \sqrt{\gamma}}{\gamma-1} r^{-\frac{\gamma-1}{2}} \sigma^{-\frac{\gamma-1}{2}}, \\
& z=u-\frac{2 k \sqrt{\gamma}}{\gamma-1} r^{-\frac{\gamma-1}{2}} \sigma^{-\frac{\gamma-1}{2}}
\end{aligned}
$$

hence (5) can be written as

$$
\begin{aligned}
& w_{t}+\mu w_{x}=-\alpha(w+z)-\frac{\gamma-1}{8 r}(w-z)(w+z)+\frac{v^{2}}{r}, \\
& z_{t}+\lambda z_{x}=-\alpha(w+z)+\frac{\gamma-1}{8 r}(w-z)(w+z)+\frac{v^{2}}{r} \\
& v_{t}=-\left(2 \alpha+\frac{w+z}{2 r}\right) v
\end{aligned}
$$

where $\lambda=-K \sqrt{\gamma} r^{-\frac{\gamma-1}{2}} \sigma^{-\frac{\gamma-1}{2}}$ and $\mu=K \sqrt{\gamma} r^{-\frac{\gamma-1}{2}} \sigma^{-\frac{\gamma-1}{2}}$ are two characteristics for (6). The corresponding initial and boundary conditions for (7) are

$$
w(x, 0)=w_{0}(x), \quad z(x, 0)=z_{0}(x), \quad v(x, 0)=v_{0}(x),
$$

and

$$
w(0, t)+z(0, t)=0
$$

In this section, we always assume the initial and boundary conditions satisfy

$$
\begin{aligned}
& w_{0}(x), z_{0}(x), v_{0}(x) \in C_{b}^{1}[0, \infty), \\
& w_{0}(x)-z_{0}(x) \geq \delta \\
& w_{0}(x)+z_{0}(x)=0, \quad \mu_{0}(0) w_{0 x}(0)=\lambda_{0}(0) z_{0 x}(0)=v^{2}(0),
\end{aligned}
$$

where $\delta$ is a positive constant and $\mu_{0}(x)$ and $\lambda_{0}(x)$ are the two given characteristics at $t=0$.

Lemma 2.1 gives the $C^{0}$ norm estimate for solutions inside the region of smooth solutions.

Lemma 2.1 [19] Under the conditions (8) and (9), if

$$
\left|v_{0}(x)\right| \leq M_{1}, \quad\left|w_{0}(x)\right| \leq M_{2}, \quad\left|z_{0}(x)\right| \leq M_{2}
$$


then when $\alpha>\frac{(\gamma-1) M_{2}+\sqrt{(\gamma-1) M_{2}^{2}+16(\gamma-1) M_{1}^{2}}}{8}$, we have

$$
|w(x, t)| \leq M_{3}, \quad|z(x, t)| \leq M_{3}, \quad|v(x, t)| \leq M_{1} e^{-\alpha t}
$$

and

$$
|w(x, t)-z(x, t)|>0,
$$

where $(x, t)$ is in the region of smooth solutions and $M_{3}=\frac{M_{1}^{2}}{2 \alpha}+M_{2}$.

The $M_{i}(i \geq 3)$ appearing in the rest of this paper are positive constants depending on $M_{1}, M_{2}$, and $\delta$.

Next we estimate the derivatives of $w(x, t), z(x, t)$ and $v(x, t)$ with respect to $x$.

Let $P(x, t)=w_{x}(x, t), Q(x, t)=z_{x}(x, t), R(x, t)=v_{x}(x, t)$. We have the following system for $P, Q$, and $R$ :

$$
\begin{aligned}
P_{t}+\mu P_{x}= & \alpha(P+Q)-K \sqrt{\gamma} \frac{\gamma+1}{\gamma-1} A r(w-z)^{\frac{2}{\gamma-1}}(P-Q) P \\
& -\frac{\gamma-1}{4 r}(w-z) P+\frac{2 v R}{r}-\frac{v^{2} \sigma}{r^{2}}-\frac{\gamma-1}{8 r}(w+z)(P-Q) \\
& -\frac{\gamma-1}{8 r}(w-z)(P+Q)+\frac{\gamma-1}{8 r^{3}}\left(\frac{\gamma-1}{4 K \sqrt{\gamma}}\right)^{-\frac{2}{\gamma-1}}(w-z)^{\frac{\gamma-3}{\gamma-1}}(w+z), \\
Q_{t}+\lambda Q_{x}= & -\alpha(P+Q)+K \sqrt{\gamma} \frac{\gamma+1}{\gamma-1} A r(w-z)^{\frac{2}{\gamma-1}}(P-Q) P \\
& +\frac{\gamma-1}{4 r}(w-z) P+\frac{2 v R}{r}-\frac{v^{2} \sigma}{r^{2}}-\frac{\gamma-1}{8 r}(w+z)(P-Q) \\
& +\frac{\gamma-1}{8 r}(w-z)(P+Q)-\frac{\gamma-1}{8 r^{3}}\left(\frac{\gamma-1}{4 K \sqrt{\gamma}}\right)^{-\frac{2}{\gamma-1}}(w-z)^{\frac{\gamma-3}{\gamma-1}}(w+z), \\
R_{t}=-\{2 \alpha+ & \left.\frac{1}{2 r}(w+z)\right\} R-\frac{1}{2 r}(P+Q) v+\frac{1}{2 r^{2}}(w+z) \sigma v,
\end{aligned}
$$

and let

$$
\begin{aligned}
& F=\frac{r}{(w-z)^{l}} P-a_{1}(w-z)^{m}-\frac{a_{2}}{r}(w-z)^{m+1}+\frac{a_{3}}{r}(w-z)^{m}(w+z), \\
& G=\frac{r}{(w-z)^{l}} Q-b_{1}(w-z)^{m}-\frac{b_{2}}{r}(w-z)^{m+1}+\frac{b_{3}}{r}(w-z)^{m}(w+z),
\end{aligned}
$$

where $A=\left(\frac{\gamma-1}{4 k \sqrt{\gamma}}\right)^{\frac{\gamma+1}{\gamma-1}}, l=-\frac{\gamma+1}{2(\gamma-1)}, m=-\frac{3-\gamma}{2(\gamma-1)}$. We have the following lemma.

Lemma 2.2 [19] Under the conditions of Lemma 2.1, if $1<\gamma<\frac{5}{3}$ or $\frac{5}{3}<\gamma<3$, and $\alpha$ being sufficiently large, there exists a constant $M_{4}$ such that if

$$
\begin{aligned}
& F(x, 0) \geq M_{4}, \\
& G(x, 0) \geq M_{4},
\end{aligned}
$$


we have

$$
\begin{aligned}
& M_{4} \leq F(x, t) \leq \sup \{F(x, 0), G(x, 0)\}, \\
& M_{4} \leq G(x, t) \leq \sup \{F(x, 0), G(x, 0)\} .
\end{aligned}
$$

Combining Lemma 2.1 and (15), we know that

$$
|P(x, t)| \leq M_{5}, \quad|Q(x, t)| \leq M_{5}, \quad|R(x, t)| \leq R_{0},
$$

where $R_{0}$ is a positive constant depending only on $M_{3},\|R(x, 0)\|_{C^{0}}$.

Remark 1 According to Lemma 2.2 and (6), we obtain $u_{x}=\frac{P+Q}{2}$, and $\rho_{x}=\frac{1}{r}+\frac{2}{\gamma-1} \times$ $\left(\frac{\gamma-1}{4 K \sqrt{\gamma}}\right) \frac{2}{\gamma-1}(w-z)^{\frac{3-\gamma}{\gamma-1}}(P-Q) r$. Then

$$
\begin{gathered}
\|u(x, t)\|_{C^{1}} \leq M_{5}, \\
\|\rho(x, t)\|_{C^{1}} \leq M_{6},
\end{gathered}
$$

and the $C^{1}$ norm estimate for solutions inside the region of smooth solutions is proved. So we get the existence of one order global smooth solution for (3) and (4).

\section{The boundedness of $C^{2}$ norm of solutions for 2D Euler equation with symmetry outside a core region}

For convenience, we list some equations which will be used later:

$$
\begin{aligned}
& \frac{d(P-Q)}{d_{\mu} t}=-2 K \sqrt{\gamma} A r(w-z)^{\frac{\gamma+1}{\gamma-1}} Q_{x}+\mathbb{A}, \\
& \frac{d(P-Q)}{d_{\lambda} t}=-2 K \sqrt{\gamma} A r(w-z)^{\frac{\gamma+1}{\gamma-1}} P_{x}+\mathbb{A}, \\
& \frac{d(P+Q)}{d_{\mu} t}=2 K \sqrt{\gamma} A r(w-z)^{\frac{\gamma+1}{\gamma-1}} Q_{x}+\mathbb{B}, \\
& \frac{d(P+Q)}{d_{\lambda} t}=2 K \sqrt{\gamma} A r(w-z)^{\frac{\gamma+1}{\gamma-1}} P_{x}+\mathbb{B},
\end{aligned}
$$

where we have used the notation $\frac{d}{d_{\mu} t}=\frac{\partial}{\partial t}+\mu \frac{\partial}{\partial x}, \frac{d}{d_{\lambda} t}=\frac{\partial}{\partial t}+\lambda \frac{\partial}{\partial x}$, and

$$
\begin{aligned}
\mathbb{A}= & -K \sqrt{\gamma} \frac{\gamma+1}{\gamma-1} \operatorname{Ar}(\omega-z)^{\frac{2}{\gamma-1}}(P-Q)(P+Q)-\frac{\gamma-1}{4 r}(w+z)(P-Q) \\
& -\frac{\gamma-1}{2 r}(w-z)(P+Q)+\frac{\gamma-1}{4 r^{3}}\left(\frac{\gamma-1}{4 K \sqrt{\gamma}}\right)^{-\frac{2}{\gamma-1}}(w-z)^{\frac{\gamma-3}{\gamma-1}}(\omega+z), \\
\mathbb{B}= & -2 \alpha(P+Q)+\frac{4 v R}{r}-\frac{2 v^{2} \sigma}{r^{2}} \\
& -K \sqrt{\gamma} \frac{\gamma+1}{\gamma-1} \operatorname{Ar}(\omega-z)^{\frac{2}{\gamma-1}}(P-Q)^{2}-\frac{\gamma-1}{4 r}(w-z)(P-Q) .
\end{aligned}
$$

Now we estimate the derivatives of $w_{x}(x, t), z_{x}(x, t)$ and $v_{x}(x, t)$ with respect to $x$. 
Let $\mathbb{H}(x, t)=w_{x x}(x, t), \mathbb{I}(x, t)=z_{x x}(x, t), \mathbb{L}(x, t)=v_{x x}(x, t)$. We have the following system for $\mathbb{H}, \mathbb{I}$, and $\mathbb{L}$ :

$$
\begin{aligned}
& \mathbb{H}_{t}+\mu \mathbb{H}_{x}=-\alpha(\mathbb{H}+\mathbb{I})-\frac{\gamma+1}{\gamma-1} K \sqrt{\gamma} \operatorname{Ar}(w-z)^{\frac{2}{\gamma-1}}(P-Q)(\mathbb{H}+\mathbb{I}) \\
& -\frac{1}{2} \frac{\gamma+1}{\gamma-1} K \sqrt{\gamma} A r(w-z)^{\frac{2}{\gamma-1}}(P+Q)(\mathbb{H}-\mathbb{I}) \\
& -6 \frac{\gamma+1}{\gamma-1} K \sqrt{\gamma} A r(w-z)^{\frac{2}{\gamma-1}}(P-Q)(\mathbb{H}-\mathbb{I}) \\
& +\frac{3(\gamma-1)}{8 r}(P-Q)(\mathbb{H}-\mathbb{I})-\frac{\gamma-1}{8 r}(P+Q)(\mathbb{H}-\mathbb{I}) \\
& -\frac{10 K^{2} \gamma}{\gamma-1}\left(\frac{\gamma-1}{4 K \sqrt{\gamma}}\right)^{\frac{2 \gamma}{\gamma-1}}(w-z)^{\frac{\gamma+1}{\gamma-1}}(\mathbb{H}-\mathbb{I}) \frac{1}{r} \\
& +\frac{2 v \mathbb{L}+2 R^{2}}{r}-\frac{2 v R \sigma+v^{2} \sigma_{x}}{r^{2}}+\frac{v^{2} \sigma^{2}}{r^{3}}+\mathbb{C}, \\
& \mathbb{I}_{t}+\lambda \mathbb{I}_{x}=-\alpha(\mathbb{H}+\mathbb{I})+\frac{\gamma+1}{\gamma-1} K \sqrt{\gamma} A r(w-z)^{\frac{2}{\gamma-1}}(P-Q)(\mathbb{H}+\mathbb{I}) \\
& +\frac{1}{2} \frac{\gamma+1}{\gamma-1} K \sqrt{\gamma} \operatorname{Ar}(w-z)^{\frac{2}{\gamma-1}}(P+Q)(\mathbb{H}-\mathbb{I}) \\
& +6 \frac{\gamma+1}{\gamma-1} K \sqrt{\gamma} A r(w-z)^{\frac{2}{\gamma-1}}(P-Q)(\mathbb{H}-\mathbb{I}) \\
& -\frac{3(\gamma-1)}{8 r}(P-Q)(\mathbb{H}-\mathbb{I})+\frac{\gamma-1}{8 r}(P+Q)(\mathbb{H}-\mathbb{I}) \\
& +\frac{10 K^{2} \gamma}{\gamma-1}\left(\frac{\gamma-1}{4 K \sqrt{\gamma}}\right)^{\frac{2 \gamma}{\gamma-1}}(w-z)^{\frac{\gamma+1}{\gamma-1}}(\mathbb{H}-\mathbb{I}) \frac{1}{r} \\
& +\frac{2 v \mathbb{L}+2 R^{2}}{r}-\frac{2 v R \sigma+v^{2} \sigma_{x}}{r^{2}}+\frac{v^{2} \sigma^{2}}{r^{3}}-\mathbb{C}, \\
& \mathbb{L}_{t}=-\left\{2 \alpha+\frac{1}{2 r}(w+z)\right\} \mathbb{L}-\frac{1}{r}(P+Q) R-\frac{1}{2 r}(\mathbb{H}+\mathbb{I}) v+\frac{1}{2 r^{2}}(w+z) \sigma R \\
& +\frac{(P+Q)}{r^{2}} v \sigma+\frac{1}{2 r^{2}}(P+Q) \sigma R+\frac{1}{2 r^{2}}(P+Q) v \sigma_{x}-\frac{1}{2 r^{3}}(w+z) \sigma^{2} v,
\end{aligned}
$$

where $1<\gamma<\frac{5}{3}$ or $\frac{5}{3}<\gamma<3$, and

$$
\begin{aligned}
\mathbb{C}= & -\frac{\gamma+1}{(\gamma-1)^{2}} K \sqrt{\gamma} A r(w-z)^{\frac{3-\gamma}{\gamma-1}}(P-Q)^{2}\{(P+Q)+4(P-Q)\}-\frac{2 \gamma-1}{4 r}(P-Q)(P+Q) \\
& -\frac{7 \gamma+5}{8 r}(P-Q)^{2}+\frac{10 K^{2} \gamma(\gamma+1)}{(\gamma-1)^{2}}\left(\frac{\gamma-1}{4 K \sqrt{\gamma}}\right)^{\frac{2 \gamma}{\gamma-1}}(w-z)^{\frac{2}{\gamma-1}}(P-Q)^{2} \frac{1}{r} \\
& +\frac{\gamma-1}{\gamma-2} K \sqrt{\gamma}\left(\frac{\gamma-1}{4 K \sqrt{\gamma}}\right)^{\frac{\gamma-3}{\gamma-1}}(w-z)^{-\frac{2}{\gamma-1}} \frac{(w+z)(P+Q)}{r^{3}} \\
& +\frac{3}{2} K \sqrt{\gamma}\left(\frac{\gamma-1}{4 K \sqrt{\gamma}}\right)^{\frac{\gamma-3}{\gamma-1}}(w-z)^{-\frac{2}{\gamma-1}} \frac{(w-z)(P+Q)}{r^{3}} \\
& -7 K \sqrt{\gamma}\left(\frac{\gamma-1}{4 K \sqrt{\gamma}}\right)^{\frac{\gamma-3}{\gamma-1}}(w-z)^{-\frac{2}{\gamma-1}} \frac{(w-z)(P-Q)}{r^{3}}
\end{aligned}
$$




$$
\begin{aligned}
& +\frac{15(\gamma-1)}{8 r^{3}}(w-z)(P-Q)+\frac{15}{r^{5}} K^{2} \gamma\left(\frac{\gamma-1}{4 K \sqrt{\gamma}}\right)^{\frac{2(\gamma-2)}{\gamma-1}}(w-z)^{\frac{2(\gamma-2)}{\gamma-1}} \\
& -\frac{3(5 \gamma-3) K \sqrt{\gamma}}{4}\left(\frac{\gamma-1}{4 K \sqrt{\gamma}}\right)^{\frac{\gamma-5}{\gamma-1}}(w-z)^{\frac{\gamma-5}{\gamma-1}} \frac{(w+z)}{r^{5}}
\end{aligned}
$$

Under the a priori estimate of $\mathbb{H}$ and $\mathbb{I}$, using the property of $P, Q, R, w, z, v$ and (24), we have

$$
|\mathbb{L}(x, t)| \leq \mathbb{L}_{0}
$$

where $\mathbb{L}_{0}$ is a positive constant depending only on $M_{3},\|\mathbb{L}(x, 0)\|_{C^{0}}$. We estimate the boundedness of $\mathbb{H}$ and $\mathbb{I}$ as follows. Let

$$
\begin{aligned}
\mathbb{H}=\overline{\mathbb{H}} & +\frac{c_{1}}{r}(\omega-z)^{s_{1}}(P-Q)+c_{2}(P-Q)^{2}(\omega-z)^{s_{2}}+c_{3}(P+Q)^{2}(\omega-z)^{s_{3}} \\
& +\frac{c_{4}}{r^{2}}(P-Q)^{2}(\omega-z)^{s_{4}}+\frac{c_{5}}{r^{2}}(P+Q)^{2}(\omega-z)^{s_{5}}+\frac{c_{6}}{r^{2}}(P-Q)(\omega-z)^{s_{6}}, \\
\mathbb{I}=\overline{\mathbb{I}} & +\frac{d_{1}}{r}(\omega-z)^{s_{1}}(P-Q)+d_{2}(P-Q)^{2}(\omega-z)^{s_{2}}+d_{3}(P+Q)^{2}(\omega-z)^{s_{3}} \\
& +\frac{d_{4}}{r^{2}}(P-Q)^{2}(\omega-z)^{s_{4}}+\frac{d_{5}}{r^{2}}(P+Q)^{2}(\omega-z)^{s_{5}}+\frac{d_{6}}{r^{2}}(P-Q)(\omega-z)^{s_{6}},
\end{aligned}
$$

where $s_{1}=s_{4}=s_{5}=-\frac{\gamma+1}{\gamma-1}<0, s_{2}=s_{3}=-1, s_{6}=0$, and let

$$
\begin{aligned}
\varepsilon\left(r, \frac{1}{r}, w, z, P, Q\right) \\
=-\frac{\gamma+1}{\gamma-1} K \sqrt{\gamma} A r(w-z)^{\frac{2}{\gamma-1}}\left(\frac{15}{2} P-\frac{13}{2} Q\right)+\frac{\gamma-1}{4 r}(P-2 Q) \\
\quad-\frac{10 K^{2} \gamma}{\gamma-1}\left(\frac{\gamma-1}{4 K \sqrt{\gamma}}\right)^{\frac{2 \gamma}{\gamma-1}}(w-z)^{\frac{\gamma+1}{\gamma-1}} \frac{1}{r} .
\end{aligned}
$$

According to (26), (27), we know that if we deduced the boundedness of $\overline{\mathbb{H}}$ and $\overline{\mathbb{I}}$, the boundedness of $\mathbb{H}$ and $\mathbb{I}$ is apparent. Comparing (26) with (22), and together with (28), we have

$$
\begin{aligned}
\overline{\mathbb{H}}_{t}+ & \mu \overline{\mathbb{H}}_{x} \\
= & \left\{-\alpha+\varepsilon\left(r, \frac{1}{r}, w, z, P, Q\right)\right\} \mathbb{I}+K \sqrt{\gamma} A r\left(4 c_{2}+\frac{5(\gamma+1)}{\gamma-1}\right)(P-Q) \mathbb{I} \\
& +\left(2 K \sqrt{\gamma} A c_{1}-\alpha\right) \mathbb{I}-K \sqrt{\gamma} A r\left(4 c_{3}-\frac{\gamma+1}{2(\gamma-1)}\right)(P+Q) \mathbb{I} \\
& +\left(4 K \sqrt{\gamma} A c_{4}-\frac{3(\gamma-1)}{8}\right) \frac{1}{r}(P-Q) \mathbb{I}+\left(\frac{\gamma-1}{8}-4 K \sqrt{\gamma} A c_{5}\right) \frac{1}{r}(P+Q) \mathbb{I} \\
& +\left(\frac{10 K^{2} \gamma}{\gamma-1}\left(\frac{\gamma-1}{4 K \sqrt{\gamma}}\right)^{\frac{2 \gamma}{\gamma-1}}+2 K \sqrt{\gamma} A c_{6}\right) \frac{1}{r}(\omega-z) \mathbb{I} \\
& -\left\{\frac{c_{1}}{r}(\omega-z)^{s_{1}}+2 c_{2}(P-Q)(\omega-z)^{s_{2}}+\frac{2 c_{4}}{r^{2}}(P-Q)(\omega-z)^{s_{4}}+\frac{c_{6}}{r^{2}}(\omega-z)^{s_{6}}\right\} \mathbb{A}
\end{aligned}
$$


Wu et al. Boundary Value Problems （2015） 2015:192

Page 8 of 13

$$
\begin{aligned}
& -\left\{2 c_{3}(P+Q)(\omega-z)^{s_{3}}+\frac{2 c_{5}}{r^{2}}(P+Q)(\omega-z)^{s_{5}}\right\} \mathbb{B}+\mathbb{C}+\mathbb{D} \\
& +\frac{2 v \mathbb{L}+2 R^{2}}{r}-\frac{2 v R \sigma+v^{2} \sigma_{x}}{r^{2}}+\frac{v^{2} \sigma^{2}}{r^{3}}
\end{aligned}
$$

and also combining (27), (23), and (28), we can obtain

$$
\begin{aligned}
& \overline{\mathbb{I}}_{t}+\lambda \overline{\mathbb{I}}_{x} \\
&=\left\{-\alpha-\varepsilon\left(r, \frac{1}{r}, w, z, P, Q\right)\right\} \mathbb{I}+K \sqrt{\gamma} A r\left(4 d_{2}-\frac{5(\gamma+1)}{\gamma-1}\right)(P-Q) \mathbb{H} \\
&+\left(2 K \sqrt{\gamma} A d_{1}-\alpha\right) \mathbb{H}+K \sqrt{\gamma} A r\left(4 d_{3}-\frac{\gamma+1}{2(\gamma-1)}\right)(P+Q) \mathbb{H} \\
&+\left(4 K \sqrt{\gamma} A d_{4}+\frac{3(\gamma-1)}{8}\right) \frac{1}{r}(P-Q) \mathbb{H}+\left(\frac{\gamma-1}{8}+4 K \sqrt{\gamma} A d_{5}\right) \frac{1}{r}(P+Q) \mathbb{H} \\
&-\left(\frac{10 K^{2} \gamma}{\gamma-1}\left(\frac{\gamma-1}{4 K \sqrt{\gamma}}\right)^{\frac{2 \gamma}{\gamma-1}}-2 K \sqrt{\gamma} A d_{6}\right) \frac{1}{r}(\omega-z) \mathbb{H} \\
&-\left\{\frac{d_{1}}{r}(\omega-z)^{s_{1}}+2 d_{2}(P-Q)(\omega-z)^{s_{2}}+\frac{2 d_{4}}{r^{2}}(P-Q)(\omega-z)^{s_{4}}+\frac{d_{6}}{r^{2}}(\omega-z)^{s_{6}}\right\} \mathbb{A} \\
&-\left\{2 d_{3}(P+Q)(\omega-z)^{s_{3}}+\frac{2 d_{5}}{r^{2}}(P+Q)(\omega-z)^{s_{5}}\right\} \mathbb{B}-\mathbb{C}+\mathbb{E} \\
&+\frac{2 v \mathbb{L}+2 R^{2}}{r}-\frac{2 v R \sigma+v^{2} \sigma_{x}}{r^{2}}+\frac{v^{2} \sigma^{2}}{r^{3}},
\end{aligned}
$$

where

$$
\begin{aligned}
\mathbb{D}= & -\left\{\frac{c_{1}}{r} s_{1}(\omega-z)^{s_{1}-1}(P-Q)+c_{2} s_{2}(P-Q)^{2}(\omega-z)^{s_{2}-1}\right. \\
& +c_{3} s_{3}(P+Q)^{2}(\omega-z)^{s_{3}-1} \\
& +\frac{c_{4}}{r^{2}} s_{4}(P-Q)^{2}(\omega-z)^{s_{4}-1}+\frac{c_{5}}{r^{2}} s_{5}(P+Q)^{2}(\omega-z)^{s_{5}-1} \\
& \left.+\frac{c_{6}}{r^{2}} s_{6}(\omega-z)^{s_{6}-1}(P-Q)\right\} \frac{d(\omega-z)}{d_{\mu} t} \\
& +\left\{\frac{c_{1}}{r^{2}}(\omega-z)^{s_{1}}(P-Q)+\frac{2 c_{4}}{r^{3}}(P-Q)^{2}(\omega-z)^{s_{4}}+\frac{2 c_{5}}{r^{3}}(P+Q)^{2}(\omega-z)^{s_{5}}\right. \\
& \left.+\frac{2 c_{6}}{r^{3}}(P-Q)(\omega-z)^{s_{6}}\right\} \frac{d r}{d_{\mu} t},
\end{aligned}
$$

and

$$
\begin{aligned}
\mathbb{E}= & -\left\{\frac{d_{1}}{r} s_{1}(\omega-z)^{s_{1}-1}(P-Q)+d_{2} s_{2}(P-Q)^{2}(\omega-z)^{s_{2}-1}+d_{3} s_{3}(P+Q)^{2}(\omega-z)^{s_{3}-1}\right. \\
& +\frac{d_{4}}{r^{2}} s_{4}(P-Q)^{2}(\omega-z)^{s_{4}-1}+\frac{d_{5}}{r^{2}} s_{5}(P+Q)^{2}(\omega-z)^{s_{5}-1} \\
& \left.+\frac{d_{6}}{r^{2}} s_{6}(\omega-z)^{s_{6}-1}(P-Q)\right\} \frac{d(\omega-z)}{d_{\mu} t}
\end{aligned}
$$




$$
\begin{aligned}
& +\left\{\frac{d_{1}}{r^{2}}(\omega-z)^{s_{1}}(P-Q)+\frac{2 d_{4}}{r^{3}}(P-Q)^{2}(\omega-z)^{s_{4}}+\frac{2 d_{5}}{r^{3}}(P+Q)^{2}(\omega-z)^{s_{5}}\right. \\
& \left.+\frac{2 d_{6}}{r^{3}}(P-Q)(\omega-z)^{s_{6}}\right\} \frac{d r}{d_{\mu} t} .
\end{aligned}
$$

In order to get the relationship between $\overline{\mathbb{H}}_{t}+\mu \overline{\mathbb{H}}_{x}$ and $\mathbb{H}$ and the relationship between $\overline{\mathbb{I}}_{t}+\lambda \overline{\mathbb{I}}_{x}$ and $\mathbb{I}$, we must choose the proper $c_{i}, d_{i}, i=1, \ldots, 6$, such that

$$
\begin{aligned}
& 2 K \sqrt{\gamma} A c_{1}-\alpha=0, \quad 4 c_{2}+\frac{5(\gamma+1)}{\gamma-1}=0, \\
& \frac{\gamma+1}{2(\gamma-1)}-4 c_{3}=0, \quad 4 K \sqrt{\gamma} A c_{4}-\frac{3(\gamma-1)}{8}=0, \\
& \frac{\gamma-1}{8}-4 K \sqrt{\gamma} A c_{5}=0, \quad \frac{10 K^{2} \gamma}{\gamma-1}\left(\frac{\gamma-1}{4 K \sqrt{\gamma}}\right)^{\frac{2 \gamma}{\gamma-1}}+2 K \sqrt{\gamma} A c_{6}=0, \\
& 2 K \sqrt{\gamma} A d_{1}-\alpha=0, \quad 4 d_{2}-\frac{5(\gamma+1)}{\gamma-1}=0, \\
& \frac{\gamma+1}{2(\gamma-1)}-4 d_{3}=0, \quad 4 K \sqrt{\gamma} A d_{4}+\frac{3(\gamma-1)}{8}=0, \\
& \frac{\gamma-1}{8}+4 K \sqrt{\gamma} A d_{5}=0, \quad \frac{10 K^{2} \gamma}{\gamma-1}\left(\frac{\gamma-1}{4 K \sqrt{\gamma}}\right)^{\frac{2 \gamma}{\gamma-1}}-2 K \sqrt{\gamma} A d_{6}=0 .
\end{aligned}
$$

This implies

$$
\begin{array}{ll}
c_{1}=d_{1}=\frac{\alpha}{2 K \sqrt{\gamma} A}, & c_{2}=-d_{2}=-\frac{5(\gamma+1)}{\gamma-1}, \\
c_{3}=d_{3}=\frac{\gamma+1}{8(\gamma-1)}, & c_{4}=-d_{4}=\frac{3(\gamma-1)}{32 K \sqrt{\gamma} A}, \\
c_{5}=-d_{5}=\frac{\gamma-1}{32 K \sqrt{\gamma} A}, & c_{6}=-d_{6}=-\frac{5}{4 A}\left(\frac{\gamma-1}{4 K \sqrt{\gamma}}\right)^{\frac{\gamma+1}{\gamma-1}} .
\end{array}
$$

Thus, the coefficient of $\mathbb{I}$ in (29) and the coefficient of $\mathbb{H}$ in (30) become zero. We can now proceed to obtain

$$
\begin{aligned}
\overline{\mathbb{H}}_{t}+\mu \overline{\mathbb{H}}_{x}= & \left\{-\alpha+\varepsilon\left(r, \frac{1}{r}, w, z, P, Q\right)\right\} \mathbb{H}-\left\{\frac{c_{1}}{r}(\omega-z)^{s_{1}}+2 c_{2}(P-Q)(\omega-z)^{s_{2}}\right. \\
& \left.+\frac{2 c_{4}}{r^{2}}(P-Q)(\omega-z)^{s_{4}}+\frac{c_{6}}{r^{2}}(\omega-z)^{s_{6}}\right\} \mathbb{A} \\
& -\left\{2 c_{3}(P+Q)(\omega-z)^{s_{3}}+\frac{2 c_{5}}{r^{2}}(P+Q)(\omega-z)^{s_{5}}\right\} \mathbb{B}+\mathbb{C}+\mathbb{D} \\
& +\frac{2 v \mathbb{L}+2 R^{2}}{r}-\frac{2 v R \sigma+v^{2} \sigma_{x}}{r^{2}}+\frac{v^{2} \sigma^{2}}{r^{3}},
\end{aligned}
$$




$$
\begin{aligned}
\overline{\mathbb{I}}_{t}+\lambda \overline{\mathbb{I}}_{x}= & \left\{-\alpha-\varepsilon\left(r, \frac{1}{r}, w, z, P, Q\right)\right\} \mathbb{I}-\left\{\frac{d_{1}}{r}(\omega-z)^{s_{1}}+2 d_{2}(P-Q)(\omega-z)^{s_{2}}\right. \\
& \left.+\frac{2 d_{4}}{r^{2}}(P-Q)(\omega-z)^{s_{4}}+\frac{d_{6}}{r^{2}}(\omega-z)^{s_{6}}\right\} \mathbb{A} \\
& -\left\{2 d_{3}(P+Q)(\omega-z)^{s_{3}}+\frac{2 d_{5}}{r^{2}}(P+Q)(\omega-z)^{s_{5}}\right\} \mathbb{B}-\mathbb{C}+\mathbb{E} \\
& +\frac{2 v \mathbb{L}+2 R^{2}}{r}-\frac{2 v R \sigma+v^{2} \sigma_{x}}{r^{2}}+\frac{v^{2} \sigma^{2}}{r^{3}} .
\end{aligned}
$$

Substituting (26) and (27) into (34) and (35), respectively, we can deduce that

$$
\begin{aligned}
\overline{\mathbb{H}}_{t}+ & \mu \overline{\mathbb{H}}_{x} \\
= & \left\{-\alpha+\varepsilon\left(r, \frac{1}{r}, w, z, P, Q\right)\right\} \overline{\mathbb{H}}+\left\{-\alpha+\varepsilon\left(r, \frac{1}{r}, w, z, P, Q\right)\right\}\left\{-\frac{c_{1}}{r}(\omega-z)^{s_{1}}(P-Q)\right. \\
& -c_{2}(P-Q)^{2}(\omega-z)^{s_{2}}-c_{3}(P+Q)^{2}(\omega-z)^{s_{3}}-\frac{c_{4}}{r^{2}}(P-Q)^{2}(\omega-z)^{s_{4}} \\
& \left.-\frac{c_{5}}{r^{2}}(P+Q)^{2}(\omega-z)^{s_{5}}-\frac{c_{6}}{r^{2}}(P-Q)(\omega-z)^{s_{6}}\right\}-\left\{\frac{c_{1}}{r}(\omega-z)^{s_{1}}+2 c_{2}(P-Q)(\omega-z)^{s_{2}}\right. \\
& \left.+\frac{2 c_{4}}{r^{2}}(P-Q)(\omega-z)^{s_{4}}+\frac{c_{6}}{r^{2}}(\omega-z)^{s_{6}}\right\} \mathbb{A}+\frac{2 v \mathbb{L}+2 R^{2}}{r}-\frac{2 v R \sigma+v^{2} \sigma_{x}}{r^{2}}+\frac{v^{2} \sigma^{2}}{r^{3}} \\
& -\left\{2 c_{3}(P+Q)(\omega-z)^{s_{3}}+\frac{2 c_{5}}{r^{2}}(P+Q)(\omega-z)^{s_{5}}\right\} \mathbb{B}+\mathbb{C}+\mathbb{D}, \\
\overline{\mathbb{I}}_{t}+ & \lambda \overline{\mathbb{I}}_{x} \\
= & \left.-\alpha-\varepsilon\left(r, \frac{1}{r}, w, z, P, Q\right)\right\} \overline{\mathbb{I}}+\left\{-\alpha-\varepsilon\left(r, \frac{1}{r}, w, z, P, Q\right)\right\}\left\{-\frac{d_{1}}{r}(\omega-z)^{s_{1}}(P-Q)\right. \\
& -d_{2}(P-Q)^{2}(\omega-z)^{s_{2}}-d_{3}(P+Q)^{2}(\omega-z)^{s_{3}}-\frac{d_{4}}{r^{2}}(P-Q)^{2}(\omega-z)^{s_{4}} \\
& \left.-\frac{d_{5}}{r^{2}}(P+Q)^{2}(\omega-z)^{s_{5}}-\frac{d_{6}}{r^{2}}(P-Q)(\omega-z)^{s_{6}}\right\} \\
& -\left\{\frac{d_{1}}{r}(\omega-z)^{s_{1}}+2 d_{2}(P-Q)(\omega-z)^{s_{2}}\right. \\
& \left.+\frac{2 d_{4}}{r^{2}}(P-Q)(\omega-z)^{s_{4}}+\frac{d_{6}}{r^{2}}(\omega-z)^{s_{6}}\right\} \mathbb{A}+\frac{2 v \mathbb{L}+2 R^{2}}{r}-\frac{2 v R \sigma+v^{2} \sigma_{x}}{r^{2}}+\frac{v^{2} \sigma^{2}}{r^{3}} \\
& -\left\{2 d_{3}(P+Q)(\omega-z)^{s_{3}}+\frac{2 d_{5}}{r^{2}}(P+Q)(\omega-z)^{s_{5}}\right\} \mathbb{B}-\mathbb{C}+\mathbb{E} .
\end{aligned}
$$

In order to simplify (36) and (37), we let

$$
\begin{aligned}
\chi_{1}= & \chi_{1}\left(r, \frac{1}{r}, w-z, w+z, P-Q, P+Q\right) \\
= & \left\{-\alpha+\varepsilon\left(r, \frac{1}{r}, w, z, P, Q\right)\right\}\left\{-\frac{c_{1}}{r}(\omega-z)^{s_{1}}(P-Q)-c_{2}(P-Q)^{2}(\omega-z)^{s_{2}}\right. \\
& -c_{3}(P+Q)^{2}(\omega-z)^{s_{3}}-\frac{c_{4}}{r^{2}}(P-Q)^{2}(\omega-z)^{s_{4}}-\frac{c_{5}}{r^{2}}(P+Q)^{2}(\omega-z)^{s_{5}} \\
& \left.-\frac{c_{6}}{r^{2}}(P-Q)(\omega-z)^{s_{6}}\right\}-\left\{\frac{c_{1}}{r}(\omega-z)^{s_{1}}+2 c_{2}(P-Q)(\omega-z)^{s_{2}}+\frac{2 c_{4}}{r^{2}}(P-Q)(\omega-z)^{s_{4}}\right.
\end{aligned}
$$




$$
\begin{aligned}
& \left.+\frac{c_{6}}{r^{2}}(\omega-z)^{s_{6}}\right\} \mathbb{A}+\frac{2 v \mathbb{L}+2 R^{2}}{r}-\frac{2 v R \sigma+v^{2} \sigma_{x}}{r^{2}}+\frac{v^{2} \sigma^{2}}{r^{3}} \\
& -\left\{2 c_{3}(P+Q)(\omega-z)^{s_{3}}+\frac{2 c_{5}}{r^{2}}(P+Q)(\omega-z)^{s_{5}}\right\} \mathbb{B}+\mathbb{C}+\mathbb{D},
\end{aligned}
$$

and

$$
\begin{aligned}
\chi_{2}= & \chi_{2}\left(r, \frac{1}{r}, w-z, w+z, P-Q, P+Q\right) \\
= & \left\{-\alpha-\varepsilon\left(r, \frac{1}{r}, w, z, P, Q\right)\right\}\left\{-\frac{d_{1}}{r}(\omega-z)^{s_{1}}(P-Q)-d_{2}(P-Q)^{2}(\omega-z)^{s_{2}}\right. \\
& -d_{3}(P+Q)^{2}(\omega-z)^{s_{3}}-\frac{d_{4}}{r^{2}}(P-Q)^{2}(\omega-z)^{s_{4}}-\frac{d_{5}}{r^{2}}(P+Q)^{2}(\omega-z)^{s_{5}} \\
& \left.-\frac{d_{6}}{r^{2}}(P-Q)(\omega-z)^{s_{6}}\right\}-\left\{\frac{d_{1}}{r}(\omega-z)^{s_{1}}+2 d_{2}(P-Q)(\omega-z)^{s_{2}}\right. \\
& \left.+\frac{2 d_{4}}{r^{2}}(P-Q)(\omega-z)^{s_{4}}+\frac{d_{6}}{r^{2}}(\omega-z)^{s_{6}}\right\} \mathbb{A}+\frac{2 v \mathbb{L}+2 R^{2}}{r}-\frac{2 v R \sigma+v^{2} \sigma_{x}}{r^{2}}+\frac{v^{2} \sigma^{2}}{r^{3}} \\
& -\left\{2 d_{3}(P+Q)(\omega-z)^{s_{3}}+\frac{2 d_{5}}{r^{2}}(P+Q)(\omega-z)^{s_{5}}\right\} \mathbb{B}-\mathbb{C}+\mathbb{E} .
\end{aligned}
$$

Using the definition of $\frac{d}{d_{\mu} t}$ and $\frac{d}{d_{\lambda} t}$, (36) and (37) become

$$
\begin{aligned}
& \frac{d \overline{\mathbb{H}}}{d_{\mu} t}=-\left\{\alpha-\varepsilon\left(r, \frac{1}{r}, w, z, P, Q\right)\right\} \overline{\mathbb{H}}+\chi_{1}\left(r, \frac{1}{r}, w-z, w+z, P-Q, P+Q\right), \\
& \frac{d \overline{\mathbb{I}}}{d_{\mu} t}=-\left\{\alpha+\varepsilon\left(r, \frac{1}{r}, w, z, P, Q\right)\right\} \overline{\mathbb{I}}+\chi_{2}\left(r, \frac{1}{r}, w-z, w+z, P-Q, P+Q\right),
\end{aligned}
$$

where $\varepsilon\left(r, \frac{1}{r}, w, z, P, Q\right)$ denotes the function depending on $r, \frac{1}{r}, w, z, P, Q$, and $\chi_{i}\left(r, \frac{1}{r}, w-\right.$ $z, w+z, P-Q, P+Q)(i=1,2)$ denotes the function depending on $r, \frac{1}{r}, w-z, w+z, P-Q$, $P+Q$, then $|\varepsilon| \leq M_{7},\left|\chi_{1}\right| \leq M_{8},\left|\chi_{2}\right| \leq M_{8}$.

The following theorem gives the boundedness of $\overline{\mathbb{H}}$ and $\overline{\mathbb{I}}$, which is defined in (26) and (27).

Theorem 3.1 Under the conditions $|P(x, t)| \leq M_{4},|Q(x, t)| \leq M_{4},|R(x, t)| \leq R_{0},|w(x, t)| \leq$ $M_{3},|z(x, t)| \leq M_{3},|v(x, t)| \leq M_{1} e^{-\alpha t}$, then when $\alpha$ is sufficiently large, we have

$$
|\overline{\mathbb{H}}(x, t)| \leq M_{9}, \quad|\overline{\mathbb{I}}(x, t)| \leq M_{9}
$$

Proof Multiplying (40) by $\overline{\mathbb{H}}$ and using the Young inequality, we obtain

$$
\begin{aligned}
\frac{1}{2} \frac{d \overline{\mathbb{H}}^{2}}{d_{\mu} t}= & -\left\{\alpha-\varepsilon\left(r, \frac{1}{r}, w, z, P, Q\right)-\frac{1}{2} \chi_{1}\left(r, \frac{1}{r}, w-z, w+z, P-Q, P+Q\right)\right\} \mathbb{H}^{2} \\
& +\frac{1}{2} \chi_{1}\left(r, \frac{1}{r}, w-z, w+z, P-Q, P+Q\right) .
\end{aligned}
$$

Referring to the proof of Theorem 2.2 in [19], we know that

$$
w(x, t)-z(x, t)>\delta_{1}>0 .
$$


Due to the boundedness of $\varepsilon$ and when $\alpha$ is sufficiently large, we can see that

$$
\alpha-\varepsilon\left(r, \frac{1}{r}, w, z, P, Q\right)-\frac{1}{2} \chi_{1}\left(r, \frac{1}{r}, w-z, w+z, P-Q, P+Q\right) \geq \frac{1}{2} \alpha>0,
$$

which implies the following:

$$
\frac{d \overline{\mathbb{H}}^{2}}{d_{\mu} t} \leq-\alpha \overline{\mathbb{H}}^{2}+\chi_{1}
$$

and using the Gronwall inequality, we have

$$
|\overline{\mathbb{H}}(x, t)|^{2} \leq M_{9}
$$

In a similar way,

$$
|\overline{\mathbb{I}}(x, t)|^{2} \leq M_{9}
$$

We have completed the proof.

\section{The existence of the second order smooth solutions of Euler equation with axial symmetry outside a core region}

The main result of this paper, which is the existence of the second order smooth solutions of this equation, is in the following theorem.

Theorem 4.1 Under the assumptions of $1<\gamma<\frac{5}{3}$ or $\frac{5}{3}<\gamma<3$ and $\alpha$ being sufficiently large, there exists second order smooth solutions of (3) and (4).

Proof According to Theorem 3.1 and (26), (27), we have $|\mathbb{H}| \leq M_{10}$, $|\mathbb{I}| \leq M_{10}$. Using (6), we obtain

$$
u_{x x}=\frac{\mathbb{H}+\mathbb{I}}{2}
$$

and

$$
\begin{aligned}
\rho_{x x}= & -\left(\frac{\gamma-1}{4 K \sqrt{\gamma}}\right)^{-\frac{2}{\gamma-1}}(w-z)^{-\frac{2}{\gamma-1}} \frac{1}{r^{2}}+\frac{2}{\gamma-1}(w-z)^{-1}(P-Q) \frac{1}{r} \\
& +\frac{2(3-\gamma)}{(\gamma-1)^{2}}\left(\frac{\gamma-1}{4 K \sqrt{\gamma}}\right)^{\frac{2}{\gamma-1}}(w-z)^{\frac{2(2-\gamma)}{\gamma-1}}(P-Q)^{2} r \\
& +\frac{2}{\gamma-1}\left(\frac{\gamma-1}{4 K \sqrt{\gamma}}\right)^{\frac{2}{\gamma-1}}(w-z)^{\frac{3-\gamma}{\gamma-1}}(\mathbb{H}-\mathbb{I}) r,
\end{aligned}
$$

because of the boundedness of $P, Q, R, w, z$, $v$, we know that

$$
\begin{gathered}
\|u\|_{C^{2}} \leq M_{10}, \\
\|\rho\|_{C^{2}} \leq M_{11} .
\end{gathered}
$$


So we get the existence of the second order smooth solution of (3) and (4). We have completed the proof.

\section{Conclusion}

In this paper, we have derived the existence of the second order smooth solutions for the 2D compressible isentropic Euler equation with axial symmetry and damping outside a core region. Compared to the transformation about (1), the proof seems much more simple. Based on the results of Lemma 2.1 and Lemma 2.2, we have obtained the boundedness of $C^{2}$ norm of the solutions. These are the improvement and innovation for the existing result in [19].

\section{Competing interests}

The authors declare that they have no competing interests.

\section{Authors' contributions}

All authors contributed equally to the writing of this paper. All authors read and approved the final manuscript.

\section{Author details}

'School of Mathematics and Statistics, Xi'an Jiaotong University, Xi'an, 710049, China. ${ }^{2}$ Institute of Mathematics, Yunnan Normal University, Kunming, 650092, China.

\section{Acknowledgements}

The work was supported by the National Natural Science Fund of China (11371289).

Received: 21 April 2015 Accepted: 10 October 2015 Published online: 23 October 2015

\section{References}

1. De Lellis, C, Szekelyhidi, LJ: On admissibility criteria for weak solutions of the Euler equations. Arch. Ration. Mech. Anal. 195(1), 225-260 (2010)

2. Zhang, T, Zheng, YX: Exact spiral solutions of the two-dimensional Euler equations. Discrete Contin. Dyn. Syst. 3, 117-133 (1997)

3. Yuen, MW: Vortical and self-similar flows of $2 \mathrm{D}$ compressible Euler equations. Commun. Nonlinear Sci. Numer. Simul. $19,2172-2180(2014)$

4. Cao, DM, Liu, ZY, Wei, JC: Regularization of point vortices pairs for the Euler equation in dimension two. Arch. Ration. Mech. Anal. 212, 179-217 (2014)

5. Pan, RG, Zhao, K: The 3D compressible Euler equations with damping in a bounded domain. J. Differ. Equ. 246, 581-596 (2009)

6. Kiselev, A, Zlatos, A: Blow up for the 2D Euler equation on some bounded domains (2014). arXiv:1406.3648

7. Yuen, MW: Some exact blowup solutions to the pressureless Euler equations in $R^{N}$. Commun. Nonlinear Sci. Numer. Simul. 16, 2993-2998 (2011)

8. Marin, M, Olivia, F: On temporal behaviour of solutions in thermoelasticity of porous micropolar bodies. An. Ştiinţ. Univ. 'Ovidius' Constanţa, Ser. Mat. 22, 169-188 (2014)

9. Douglis, A: Existence theorems for hyperbolic systems. Commun. Pure Appl. Math. 5, 119-154 (1952)

10. Hoff, D: Global smooth solutions to quasilinear hyperbolic systems in diagonal from. J. Math. Anal. Appl. 86, 221-238 (1982)

11. Ling, H, Li, T: Global smooth solution of Cauchy problems for a class of quasilinear hyperbolic systems. Chin. Ann. Math. 4B, 109-115 (1983)

12. John, F: Formation of singularities in one-dimensional nonlinear wave propagation. Commun. Pure Appl. Math. 27, 377-405 (1974)

13. Lax, PD: Development of singularities of solutions of nonlinear hyperbolic partial differential equations. J. Math. Phys. 5, 611-613 (1964)

14. Li, T, Yu, WC: Boundary Value Problems for Quasilinear Hyperbolic System. Duke University Press, Durham (1985)

15. Lin, LW, Yang, T: Existence and nonexistence of global smooth solutions for damped p-system with "really large" initial data. J. Partial Differ. Equ. 4, 45-51 (1991)

16. Liu, TP: Development of singularities in the nonlinear waves for quasilinear hyperbolic partial differential equations. J. Differ. Equ. 33, 92-111 (1979)

17. Nishida, T: Nonlinear hyperbolic equations and related topics in fluid dynamics. Publications Mathematiques D'ocsay 78.02, Department de Mathematique, Paris-Sud (1978)

18. Zhu, CJ: Global resolvability for a viscoelastic model with relaxation. Proc. R. Soc. Edinb. 25A, 1277-1285 (1995)

19. Ying, LA, Yang, T, Zhu, CJ: Existence of global smooth solutions for Euler equations with symmetry. Commun. Partial Differ. Equ. 22(7), 1277-1285 (1997)

20. Hou, YT, Zhen, L, Luo, G, Wang, S, Chen, Z: On finite time singularity and regularity of axisymmetric model for the 3D Euler equation. Arch. Ration. Mech. Anal. 212, 683-706 (2014) 